\title{
RAMSEY NUMBERS FOR BIPARTITE GRAPHS WITH SMALL BANDWIDTH
}

\author{
GUILHERME O. MOTA, GÁBOR N. SÁRKÖZY, MATHIAS SCHACHT, AND ANUSCH TARAZ
}

\begin{abstract}
We estimate Ramsey numbers for bipartite graphs with small bandwidth and bounded maximum degree. In particular we determine asymptotically the two and three color Ramsey numbers for grid graphs. More generally, we determine asymptotically the two color Ramsey number for bipartite graphs with small bandwidth and bounded maximum degree and the three color Ramsey number for such graphs with the additional assumption that the bipartite graph is balanced.
\end{abstract}

\section{§1. INTRODUCTION}

For graphs $G_{1}, \ldots, G_{r}$, the Ramsey number $R\left(G_{1}, \ldots, G_{r}\right)$ is the smallest integer $n$ such that if the edges of a complete graph $K_{n}$ are partitioned into $r$ disjoint color classes giving $r$ graphs $H_{1}, \ldots, H_{r}$, then at least one $H_{i}(1 \leqslant i \leqslant r)$ contains a subgraph isomorphic to $G_{i}$. The existence of such an integer follows from Ramsey's theorem. The number $R\left(G_{1}, \ldots, G_{r}\right)$ is called the Ramsey number of the graphs $G_{1}, \ldots, G_{r}$. Determining $R\left(G_{1}, G_{2}, \ldots, G_{r}\right)$ for general graphs appears to be a difficult problem (see, e.g., [9] or [19]). For $r=2$, a well-known theorem of Gerencsér and Gyárfás [8] states that

$$
R\left(P_{n}, P_{n}\right)=\left\lfloor\frac{3 n-2}{2}\right\rfloor,
$$

where $P_{n}$ denotes the path with $n \geqslant 2$ vertices. In [13] more general trees were considered. For a tree $T$, we write $t_{1}$ and $t_{2}$, with $t_{2} \geqslant t_{1}$, for the sizes of the vertex classes of $T$ as a bipartite graph. Note that $R(T, T) \geqslant 2 t_{1}+t_{2}-1$, since the following edge-coloring of $K_{2 t_{1}+t_{2}-2}$ has no monochromatic copy of $T$. Partition the vertices into two classes $V_{1}$ and $V_{2}$ such that $\left|V_{1}\right|=t_{1}-1$ and $\left|V_{2}\right|=t_{1}+t_{2}-1$, then use color "red" for all edges inside the two classes and use color "blue" for all edges between the classes. A similar

2010 Mathematics Subject Classification. 05C55 (primary), 05C38 (secondary).

Key words and phrases. Ramsey theory, bandwidth, regularity lemma.

G. O. Mota was supported by FAPESP (2009/06294-0 and 2012/00036-2) and he gratefully acknowledges the support of NUMEC/USP, Project MaCLinC/USP. G.N. Sárközy was supported in part by the NSF Grant DMS-0968699 and by OTKA Grant K104373. M. Schacht was supported by the HeisenbergProgramme of the DFG (grant SCHA 1263/4-1). A. Taraz was supported in part by DFG grant TA 309/22. The cooperation was supported by a joint CAPES-DAAD project (415/ppp-probral/po/D08/11629, Proj. no. 333/09). 
edge-coloring of $K_{2 t_{2}-2}$ with two classes both of size $t_{2}-1$ shows that $R(T, T) \geqslant 2 t_{2}-1$. Thus

$$
R(T, T) \geqslant \max \left\{2 t_{1}+t_{2}, 2 t_{2}\right\}-1
$$

Haxell, Łuczak and Tingley provided in [13] an asymptotically matching upper bound for trees $T$ with $\Delta(T)=o\left(t_{2}\right)$.

We partially extend this to bipartite graphs with small bandwidth and a more restrictive maximum degree condition. A graph $H=\left(W, E_{H}\right)$ is said to have bandwidth at most $b$, if there exists a labelling of the vertices by numbers $1, \ldots, n$ such that for every edge $i j \in E_{H}$ we have $|i-j| \leqslant b$. We focus our attention on the following class of bipartite graphs.

Definition 1.1. A bipartite graph $H$ is a $(\beta, \Delta)$-graph if its bandwidth is at most $\beta|V(H)|$ and its maximum degree is at most $\Delta$. We say that $H$ is a balanced $(\beta, \Delta)$-graph if it has a proper 2-coloring $\chi: V(H) \rightarrow[2]$ such that ||$\chi^{-1}(1)|-| \chi^{-1}(2)|| \leqslant \beta\left|\chi^{-1}(2)\right|$.

For example, it was shown in [5] that sufficiently large planar graphs with maximum degree at most $\Delta$ are $(\beta, \Delta)$-graphs for any fixed $\beta>0$. Our first theorem is an analogue of the result in [13] for $(\beta, \Delta)$-graphs.

Theorem 1.2. For every $\gamma>0$ and natural number $\Delta$, there exist a constant $\beta>0$ and natural number $n_{0}$ such that for every $(\beta, \Delta)$-graph $H$ on $n \geqslant n_{0}$ vertices with a proper 2-coloring $\chi: V(H) \rightarrow[2]$ where $t_{1}=\left|\chi^{-1}(1)\right|$ and $t_{2}=\left|\chi^{-1}(2)\right|$, with $t_{1} \leqslant t_{2}$, we have

$$
R(H, H) \leqslant(1+\gamma) \max \left\{2 t_{1}+t_{2}, 2 t_{2}\right\}
$$

For more recent results on the Ramsey number of graphs of higher chromatic number and sublinear bandwidth, we refer the reader to the work of Allen, Brightwell and Skokan [1].

For $r \geqslant 3$ colors less is known about Ramsey numbers. Let $T$ be a tree and consider $t_{1}$ and $t_{2}$, with $t_{1} \leqslant t_{2}$, the sizes of the vertex classes of $T$ as a bipartite graph. For $r=3$ colors the following construction gives a lower bound for $R(T, T, T)$. Partition the vertices of $K_{t_{1}+3 t_{2}-3}$ into four classes, one special class $V_{0}$ with $\left|V_{0}\right|=t_{1}$ and three classes $V_{1}, V_{2}$ and $V_{3}$ of size $t_{2}-1$. The color for edges inside $V_{0}$ is arbitrary. Use color $i$ inside the classes $V_{i}$ and color $i$ between $V_{i}$ and $V_{0}$ for $1 \leqslant i \leqslant 3$. Finally, use color $k \in[3] \backslash\{i, j\}$ for edges between the classes $V_{i}$ and $V_{j}$ for $1 \leqslant i<j \leqslant 3$. It is easy to check that this coloring yields no monochromatic copy of $T$. Thus

$$
R(T, T, T) \geqslant t_{1}+3 t_{2}-2
$$


Proving a conjecture of Faudree and Schelp [6], it was shown in [10] that this construction is optimal for large paths, i.e., for sufficiently large $n$ we have

$$
R\left(P_{n}, P_{n}, P_{n}\right)=\left\{\begin{array}{l}
2 n-1 \text { for odd } n \\
2 n-2 \text { for even } n
\end{array}\right.
$$

Asymptotically this was also proved independently by Figaj and Łuczak [7]. Benevides and Skokan [2] proved that $R\left(C_{n}, C_{n}, C_{n}\right)=2 n$ for sufficiently large even $n$. Our second result extend the two above ones asymptotically to balanced $(\beta, \Delta)$-graphs.

Theorem 1.3. For every $\gamma>0$ and every natural number $\Delta$, there exist a constant $\beta>0$ and natural number $n_{0}$ such that for every balanced $(\beta, \Delta)$-graph $H$ on $n \geqslant n_{0}$ vertices we have

$$
R(H, H, H) \leqslant(2+\gamma) n
$$

In particular, Theorems 1.2 and 1.3 give the asymptotics for two and three color Ramsey numbers of grid graphs. The 2-dimensional grid graph $G_{a, b}$ is the graph with vertex set $V=[a] \times[b]$ and there is an edge between two vertices if they are equal in one coordinate and consecutive in the other. Note that any grid graph $G_{a, b}$ on $a b$ vertices has bandwidth at most $\min \{a, b\}$ and satisfies $\Delta(G) \leqslant 4$. Moreover, $G_{a, b}$ is a balanced $(\beta, 4)$-graph for any fixed $\beta>0$ and sufficiently large $a b$. Consequently, Theorems 1.2 and 1.3 combined with (1) and (2) give the following corollary.

Corollary 1.4. For grid graphs $G_{a, b}$ we have

$$
R\left(G_{a, b}, G_{a, b}\right)=(3 / 2+o(1)) a b
$$

and

$$
R\left(G_{a, b}, G_{a, b}, G_{a, b}\right)=(2+o(1)) a b,
$$

where $o(1)$ tends to 0 as $a b \rightarrow \infty$.

We remark that similar bounds follow for bipartite planar graphs with bounded degree and grids of higher dimension.

This paper is organized as follows. We first give the necessary tools in Section 2 and then present a detailed proof of Theorem 1.3 in Section 3. The proof of Theorem 1.2 is very similar and here we only present an outline, in Section 4. 


\section{§2. Auxiliary RESUlts}

The main purpose of this section is to present the tools for the proof of Theorem 1.3. A main tool in the proof is Szemerédi's Regularity Lemma [22]. We discuss the Regularity Method in Section 2.1. In Sections 2.2 and 2.3 we give some results that allow us to make use of the regularity method.

2.1. The Regularity Method. Given an graph $G$ on $n$ vertices, the density of $G$ is given by $d_{G}=e(G) /\left(\begin{array}{l}n \\ 2\end{array}\right)$. Furthermore, if $A, B \subset V(G)$ are non-empty and disjoint, we denote by $e_{G}(A, B)$ the number of edges of $G$ with one endpoint in $A$ and the other in $B$ and

$$
d_{G}(A, B)=\frac{e_{G}(A, B)}{|A||B|}
$$

is the density of $G$ between $A$ and $B$.

The bipartite graph $G=(A, B ; E)$ is called $\varepsilon$-regular if for all $X \subset A, Y \subset B$ with $|X|>\varepsilon|A|$ and $|Y|>\varepsilon|B|$ we have

$$
\left|d_{G}(X, Y)-d_{G}(A, B)\right|<\varepsilon
$$

We say that $G$ is $(\varepsilon, d)$-regular if it is $\varepsilon$-regular and $d_{G}(A, B) \geqslant d$. An $\varepsilon$-regular bipartite graph $(A, B ; E)$ is called $(\varepsilon, d)$-super-regular if we have $\operatorname{deg}_{G}(a)>d|B|$ for all $a \in A$ and also $\operatorname{deg}_{G}(b)>d|A|$ for all $b \in B$.

For a graph $G=(V, E)$, a partition $\left(V_{i}\right)_{i \in[s]}$ of $V$ is said to be $(\varepsilon, d)$-regular (resp. super-regular) on a graph $R$ with vertex set contained in $[s]$ if the bipartite subgraph of $G$ induced by the pair $\left\{V_{i}, V_{j}\right\}$ is $(\varepsilon, d)$-regular (resp. super-regular) whenever $i j \in E(R)$. We say that a graph $R$ on vertex set $[s]$ is the $(\varepsilon, d)$-reduced graph of $\left(V_{i}\right)_{i \in[s]}$ (or of $G$ ) if $i j$ is an edge of $R$ if and only if the bipartite graph defined by the pair $\left\{V_{i}, V_{j}\right\}$ is $(\varepsilon, d)$ regular in $G$. The proof of Theorem 1.3 is based on the following three color version of the Regularity Lemma.

Lemma 2.1 (Regularity Lemma). For every $\varepsilon>0$ and every integer $k_{0}>0$ there is a positive integer $K_{0}\left(\varepsilon, k_{0}\right)$ such that for $n \geqslant K_{0}$ the following holds. For all graphs $G_{1}, G_{2}$ and $G_{3}$ with $V\left(G_{1}\right)=V\left(G_{2}\right)=V\left(G_{3}\right)=V,|V|=n$, there is a partition of $V$ into $k+1$ classes $V=V_{0}, V_{1}, V_{2}, \ldots, V_{k}$ such that

(i) $k_{0} \leqslant k \leqslant K_{0}$

(ii) $\left|V_{1}\right|=\left|V_{2}\right|=\cdots=\left|V_{k}\right|$,

(iii) $\left|V_{0}\right|<\varepsilon n$

(iv) apart from up to at most $\varepsilon\left(\begin{array}{c}k \\ 2\end{array}\right)$ exceptional pairs, the pairs $\left\{V_{i}, V_{j}\right\}$ are $\varepsilon$-regular in $G_{1}, G_{2}$ and $G_{3}$. 
For extensive surveys on the Regularity Lemma and its applications see $[14,17]$. A key component of the regularity method is the Blow-up Lemma [15] (see also [16, 20,21] for alternative proofs). This lemma guarantees that bipartite spanning subgraphs of bounded degree can be embedded into super-regular pairs. In fact, the statement is more general and allows the embedding of $r$-chromatic graphs into the union of $r$ vertex classes that form $\left(\begin{array}{l}r \\ 2\end{array}\right)$ super-regular pairs.

Here we will use a version of the Blow-up lemma that allows us to embed graphs $H$ of bounded-degree in a graph $G$ when $G$ and $H$ have "compatible" partitions, in the sense explained in the definition below. In our proof we will embed $H$ in parts, considering a partition of a monochromatic subgraph $G$ of $K_{N}$ with corresponding reduced graph containing a tree $T$ that contains a "large" matching $M$, where the bipartite subgraphs of $G$ corresponding to the matching edges are super-regular pairs.

Definition 2.2. Suppose $H=\left(W, E_{H}\right)$ is a graph, $T=\left([s], E_{T}\right)$ is a tree, and $M=\left([s], E_{M}\right)$ is a subgraph of $T$ where $E_{M}$ is a matching. Given a partition $\left(W_{i}\right)_{i \in[s]}$ of $W$, let $U_{i}$, for $i \in[s]$, be the set of vertices in $W_{i}$, with neighbors in some $W_{j}$ with ij $\in E_{T} \backslash E_{M}$ and set $U=\bigcup U_{i}$ and $U_{i}^{\prime}=N_{H}(U) \cap\left(W_{i} \backslash U\right)$.

We say that $\left(W_{i}\right)_{i \in[s]}$ is $(\varepsilon, T, M)$-compatible with a vertex partition $\left(V_{i}\right)_{i \in[s]}$ of some graph $G=(V, E)$ if the following holds:

(I) $x y \in E_{H}$ for $x \in W_{i}$ and $y \in W_{j}$ implies $i j \in E_{T}$ for all $i, j \in[s]$,

(II) $\left|W_{i}\right| \leqslant\left|V_{i}\right|$ for all $i \in[s]$,

(III) $\left|U_{i}\right| \leqslant \varepsilon\left|V_{i}\right|$ for all $i \in[s]$,

(IV) $\left|U_{i}^{\prime}\right|,\left|U_{j}^{\prime}\right| \leqslant \varepsilon \min \left\{\left|V_{i}\right|,\left|V_{j}\right|\right\}$ for all $i j \in E_{M}$.

We remark that for connected graphs $H$ and for every vertex $i$ of $T$ which is not covered by $M$ we have $U_{i}=W_{i}$ and $U_{i}^{\prime}=\varnothing$.

The following corollary of the Blow-up Lemma (see [3]) asserts that in the setup of Definition 2.2 graphs $H$ of bounded degree can be embedded into $G$, if $G$ admits a partition being sufficiently regular on $T$ and super-regular on $M$.

Lemma 2.3 (Embedding Lemma [3,4]). For all $d, \Delta>0$ there is a $\varepsilon=\varepsilon(d, \Delta)>0$ such that the following holds.

Let $G=(V, E)$ be an $N$-vertex graph that has a partition $\left(V_{i}\right)_{i \in[s]}$ of $V$ with $(\varepsilon, d)$-reduced graph $T$ on $[s]$ which is $(\varepsilon, d)$-super-regular on a graph $M \subset T$. Further, let $H=\left(W, E_{H}\right)$ be an $n$-vertex graph with maximum degree $\Delta(H) \leqslant \Delta$ and $n \leqslant N$ that has a vertex partition $\left(W_{i}\right)_{i \in[s]}$ of $W$ which is $(\varepsilon, T, M)$-compatible with $\left(V_{i}\right)_{i \in[s]}$. Then $H \subset G$. 
We close this section with two simple facts. They follow easily from the definitions of regular and super-regular pairs.

Fact 2.4. Let $B=\left(V_{1}, V_{2} ; E\right)$ be an $\varepsilon$-regular bipartite graph, let $\alpha>\varepsilon$, and let $V_{1}^{\prime} \subset V_{1}$ and $V_{2}^{\prime} \subset V_{2}$ be subsets with $\left|V_{1}^{\prime}\right| \geqslant \alpha\left|V_{1}\right|$ and $\left|V_{2}^{\prime}\right| \geqslant \alpha\left|V_{2}\right|$. Then for $\varepsilon^{\prime}=\max \{\varepsilon / \alpha, 2 \varepsilon\}$ the graph $B^{\prime}=\left(V_{1}^{\prime}, V_{2}^{\prime} ; E_{B}\left(V_{1}^{\prime}, V_{2}^{\prime}\right)\right)$ is $\varepsilon^{\prime}$-regular with $\left|d_{B}\left(V_{1}, V_{2}\right)-d_{B^{\prime}}\left(V_{1}^{\prime}, V_{2}^{\prime}\right)\right|<\varepsilon$.

Fact 2.5. Consider a graph $G=(V, E)$ with an $(\varepsilon, d)$-regular partition $\left(V_{i}\right)_{i \in[s]}$ of $V$ with $\left|V_{i}\right|=m$ for $1 \leqslant i \leqslant s$. Let $T$ be a graph on vertex set $[s]$ contained in the corresponding $(\varepsilon, d)$-reduced graph of $\left(V_{i}\right)_{i \in[s]}$ and let $M$ be a matching contained in $T$. Then for each vertex $i$ of $M$, the associated set $V_{i}$ in $G$ contains a subset $V_{i}^{\prime}$ of size $(1-\varepsilon r) m$ such that for every edge ij of $M$ the bipartite graph $\left(V_{i}^{\prime}, V_{j}^{\prime} ; E_{G}\left(V_{i}^{\prime}, V_{j}^{\prime}\right)\right)$ is $(\varepsilon /(1-\varepsilon r), d-(1+r) \varepsilon)$ super-regular.

2.2. Regular blow-up of a tree. In this section we show, in Lemma 2.8, that for any coloring of $E\left(K_{N}\right)$ there exists a dense, regular, monochromatic subgraph of $K_{N}$ with some structural properties that allow us to embed $H$ into this subgraph. Here the notion of a connected matching in the reduced graph (originating in [18], see also [7,10-12]) plays a central role. A connected matching in a graph $R$ is a matching $M$ such that all edges of $M$ are in the same connected component of $R$. The following lemma, proved in [7], states that in a 3-colored almost complete graph we can always find a connected matching that covers almost half of the vertices and it is contained in a monochromatic tree.

Lemma 2.6. For every $\delta>0$ there exist an $\varepsilon_{0}>0$ and a natural number $k_{0}$ such that for every $\varepsilon<\varepsilon_{0}$ and $k \geqslant k_{0}$ and for every 3-edge colored graph $R$ on $k$ vertices with density at least $(1-\varepsilon)$ there exists a matching $M$ with at least $(1-\delta) k / 4$ edges in $R$ that is contained in a monochromatic tree $T \subset R$.

This lemma can be found in a stronger structural form in [10]. In fact, there it is proved that either there is a monochromatic connected matching covering more than half of the vertices, or the graph $R$ is close to one of two extremal cases. It is not hard to see that in both extremal cases there is a monochromatic connected matching $M$ of size at least $(1-\delta)|V(R)| / 4$. We will also make use of the following simple fact.

Fact 2.7. If a tree $T$ contains a matching $M$ with $\ell$ edges then the vertices covered by the matching can be labelled in such way that $E_{M}=\left\{x_{i} y_{i}: i=1, \ldots, \ell\right\}$ and $x_{i}$ and $x_{j}$ are at an even distance in $T$ for all $1 \leqslant i<j \leqslant \ell$.

Indeed, consider a proper two-coloring $\chi: V(T) \rightarrow[2]$. Label those endpoints of the matching edges with $x_{i}$ that are in $\chi^{-1}(1)$ and label the other endpoints by $y_{i}$. Clearly, the distance in $T$ between any $x_{i}$ and $x_{j}$ is even, since they belong to the same color class. 
Given a coloring $\chi_{K_{N}}: E\left(K_{n}\right) \rightarrow[3]$, we denote by $G_{1}$ the spanning subgraph of $K_{n}$ such that $i j \in E\left(G_{1}\right)$ if and only if $\chi_{K_{N}}(i j)=1$.

Lemma 2.8. For every $\gamma>0$ there exists an $\varepsilon_{0}>0$ such that for every $\varepsilon \in\left(0, \varepsilon_{0}\right)$, there exists a natural number $K_{0}$ such that for all $N=(2+\gamma) n \geqslant K_{0}$ and for every coloring $\chi_{K_{N}}: E\left(K_{N}\right) \rightarrow[3]$, there exist a color (say color 1 ), integers $\ell, \ell^{\prime}, k$ with $\ell, \ell^{\prime} \leqslant k \leqslant K_{0}$ and $\ell \geqslant(1-\gamma / 4) k / 4$, a tree $T$ on vertex set $\left\{x_{1}, \ldots, x_{\ell}, y_{1}, \ldots, y_{\ell}, z_{1}, \ldots, z_{\ell^{\prime}}\right\}$ containing a matching $M$ with edge set $E_{M}=\left\{x_{i} y_{i}: i=1, \ldots, \ell\right\}$ with an even distance in $T$ between any $x_{i}$ and $x_{j}$ for all $i$ and $j$, such that there exists a partition $\left(V_{i}\right)_{i \in[k]}$ of $V\left(K_{N}\right)$ such that $G_{1}$ is $(\varepsilon, 1 / 3)$-regular on $T$ and $\left|V_{1}\right|=\ldots=\left|V_{k}\right| \geqslant(1-\varepsilon) N / k$.

Proof. Fix $\gamma>0$ and set $\delta=\gamma / 4$. Let $\varepsilon_{0}$ and $k_{0}$ be the constants obtained from Lemma 2.6 applied with $\delta$. Fix $\varepsilon<\varepsilon_{0}$ and let $K_{0}$ be obtained by an application of the Regularity Lemma (Lemma 2.1) with parameters $\varepsilon$ and $k_{0}$. Finally let $N=(2+\gamma) n \geqslant K_{0}$ be given.

Consider an arbitrary 3-coloring $\chi_{K_{N}}: E\left(K_{N}\right) \rightarrow$ [3] of the edges of $K_{N}$ and spanning subgraphs $G_{1}, G_{2}$ and $G_{3}$ of $K_{N}$ where $i j \in G_{s}$ if and only if $\chi_{K_{N}}(i j)=s$, for $s=1,2,3$. Owing to the Regularity Lemma, there is a partition $V_{0}, V_{1}, \ldots, V_{k}$ of the vertices of $K_{N}$ such that $\left|V_{i}\right|=m \geqslant(1-\varepsilon) N / k$ for $1 \leqslant i \leqslant k$ and more than $(1-\varepsilon)\left(\begin{array}{l}k \\ 2\end{array}\right)$ pairs $\left\{V_{i}, V_{j}\right\}$ for $1 \leqslant i<j \leqslant k$ are $\varepsilon$-regular in $G_{1}, G_{2}$ and $G_{3}$, where $k_{0} \leqslant k \leqslant K_{0}$.

We define the following reduced graph: let $R$ be the graph with vertex set $[k]$, which contains the edge $i j$ if and only if $\left\{V_{i}, V_{j}\right\}$ is $\varepsilon$-regular in each of $G_{1}, G_{2}$ and $G_{3}$. Thus, $|E(R)| \geqslant(1-\varepsilon)\left(\begin{array}{l}k \\ 2\end{array}\right)$. We know that $R$ is a graph on $k$ vertices with density at least $(1-\varepsilon)$. Now we define a coloring $\chi_{R}: E(R) \rightarrow$ [3] of the edges of $R$ such that $\chi_{R}(i, j)=s$ if $s \in[3]$ is the biggest integer in [3] such that $\left|E_{G_{s}}\left(V_{i}, V_{j}\right)\right| \geqslant\left|E_{G_{r}}\left(V_{i}, V_{j}\right)\right|$ for $1 \leqslant r \leqslant 3$, i.e., the edge $i j$ receives one of the colors that appears in most edges of $E_{K_{N}}\left(V_{i}, V_{j}\right)$ with respect to the coloring $\chi_{K_{N}}$ of $E\left(K_{N}\right)$. If $\chi_{R}(i j)=s$, then $\left|E_{G_{s}}\left(V_{i}, V_{j}\right)\right| \geqslant\left|V_{i}\right|\left|V_{j}\right| / 3$.

Since $k \geqslant k_{0}$ and the density of $R$ is at least $(1-\varepsilon)$, by Lemma 2.6, we know that $R$ contains a monochromatic tree $T$ that contains a matching $M$ of size $\ell \geqslant(1-\delta) k / 4$. Without loss of generality we may assume that the edges of $T$ are colored with color 1 . By Fact 2.7 we can label $M=\left(\left\{x_{i}, y_{i}\right\}\right)_{i}$ such that $x_{i}$ and $x_{j}$ are at even distance in $T$ for every $1 \leqslant i<j \leqslant \ell$.

Let $\left\{z_{1}, \ldots, z_{\ell^{\prime}}\right\}$ be the vertices of $T$ that are not covered by edges of the matching $M$. Since all the edges of $T$ are present in $R$ we know that, for all $i j \in E(T)$, the pairs $\left\{V_{i}, V_{j}\right\}$ are $\varepsilon$-regular in $G_{1}$ with $\left|E_{G_{1}}\left(V_{i}, V_{j}\right)\right| \geqslant\left|V_{i}\right|\left|V_{j}\right| / 3$. Thus we are done, since we can consider the graph composed of the classes $V_{i}$ for every $i \in V(T)$ and with edge set $E_{G_{1}}\left(V_{i}, V_{j}\right)$ between every pair. 
2.3. Balanced intervals. By definition, given $\beta>0$ and a natural $\Delta$, a balanced $(\beta, \Delta)$ graph $H$ has a 2-coloring of its vertices that uses both colors similarly often in total, but this does not have to be true locally. In this section we show how to balance $H$ so that the two colors appear in approximately the same number of vertices also locally.

Given a graph $H=(W, E)$ with $W=\left\{w_{1}, \ldots, w_{n}\right\}$, let $\chi: W \rightarrow[2]$ be a 2-coloring. Define the function $C_{i}$ such that if $W^{\prime} \subset W$ then, for $i=1,2$, we have $C_{i}\left(W^{\prime}\right)=\left|\chi^{-1}(i) \cap W^{\prime}\right|$. We say that $\chi$ is a $\beta$-balanced coloring of $W$ if $1-\beta \leqslant C_{1}(W) / C_{2}(W) \leqslant 1+\beta$. A subset $I \subset W$ is called interval if there exists $p<q$ such that $I=\left\{w_{p}, w_{p+1}, \ldots, w_{q}\right\}$. Finally, let $\ell^{\prime}$ and $\hat{\ell}$ be positive integers with $\ell^{\prime} \leqslant \hat{\ell}$ and let $\sigma:\left[\ell^{\prime}\right] \rightarrow[\hat{\ell}]$ be an injection. Consider a partition of $W$ into a set of intervals $\mathcal{I}=\left\{I_{1}, \ldots, I_{\hat{\ell}}\right\}$. We define $C_{i}(\mathcal{I}, \sigma, a)=\sum_{j=1}^{a} C_{i}\left(I_{\sigma(j)}\right)$ for $i=1,2$. If it is clear what partition we are considering then we write $C_{i}(\sigma, a)$ for simplicity.

Given a graph $H=(W, E)$, let $c: W \rightarrow[2]$ be a coloring of $W$ such that $H$ is globally balanced. Roughly speaking, the next lemma states that every partition of $W$ into intervals of almost the same size can be rearranged in some way that, after the rearrangement, if we remove the "last" intervals, then, in the subgraph of $H$ induced by the remaining vertices, the difference between the number of vertices $w$ with $c(w)=1$ and those $w$ with $c(w)=2$ is "small".

Lemma 2.9. For every integer $\hat{\ell} \geqslant 1$ there exists $n_{0}$ such that if $H=(W, E)$ is a graph with $W=\left\{w_{1}, \ldots, w_{n}\right\}$ with $n \geqslant n_{0}$, then every $\beta$-balanced 2 -coloring $\chi$ of $W$ with $\beta \leqslant 2 / \hat{\ell}$, and every partition of $W$ into intervals $I_{1}, \ldots, I_{\hat{\ell}}$ with sizes $\left|I_{1}\right| \leqslant \ldots \leqslant\left|I_{\hat{\ell}}\right| \leqslant\left|I_{1}\right|+1$ there exists a permutation $\sigma:[\hat{\ell}] \rightarrow[\hat{\ell}]$ such that for every $1 \leqslant i \leqslant \hat{\ell}$ we have

$$
\left|C_{1}(\sigma, i)-C_{2}(\sigma, i)\right| \leqslant \frac{n}{\hat{\ell}}+1
$$

Proof. Fix $\hat{\ell} \geqslant 1$ and set $n_{0}=2 \hat{\ell}^{3}$. Let $H=(W, E)$ be a graph such that $W=\left\{w_{1}, \ldots, w_{n}\right\}$ with $n \geqslant n_{0}$. Fix a $\beta$-balanced coloring $\chi$ of $W$ and a partition of $W$ into intervals $I_{1}, \ldots, I_{\hat{\ell}}$ with $\left|I_{1}\right| \leqslant \ldots \leqslant\left|I_{\hat{\ell}}\right| \leqslant\left|I_{1}\right|+1$ where $\beta \leqslant 2 / \hat{\ell}$.

Let us construct the permutation $\sigma$ iteratively. We can take any integer on $[\hat{\ell}]$ to be $\sigma(1)$, because the size of the intervals is at most $n / \hat{\ell}+1$. Suppose $\sigma(1), \ldots, \sigma(i)$ were defined in such a way that $\left|C_{1}(\sigma, i)-C_{2}(\sigma, i)\right| \leqslant n / \hat{\ell}+1$, where $i \leqslant \hat{\ell}-1$.

If $C_{1}(\sigma, i)=C_{2}(\sigma, i)$, then clearly $\sigma(i+1)$ can be defined as being any of the remaining integers on $[\hat{\ell}]$. So, w.l.o.g. assume that $C_{1}(\sigma, i)=C_{2}(\sigma, i)+k$, with $1 \leqslant k \leqslant n / \hat{\ell}+1$. But since $C_{1}(\sigma, i)+C_{2}(\sigma, i) \leqslant i(n / \hat{\ell}+1)$, we can conclude that

$$
C_{2}(\sigma, i) \leqslant \frac{i n}{2 \hat{\ell}}-\frac{k-i}{2} .
$$


We will prove that there exists some $r \in[\hat{\ell}] \backslash \bigcup_{j=1}^{i} \sigma(j)$ with $C_{2}\left(I_{r}\right) \geqslant k / 2$. Suppose by contradiction that $C_{2}\left(I_{r}\right)<k / 2$ for all integers $r \in[\hat{\ell}] \backslash \bigcup_{j=1}^{i} \sigma(j)$. This fact together with (3) implies the following.

$$
\begin{aligned}
C_{2}(W) & \leqslant C_{2}(\sigma, i)+(\hat{\ell}-i) \frac{k}{2}=\frac{i n}{2 \hat{\ell}}+(\hat{\ell}-i-1) \frac{k}{2}+\frac{i}{2} \\
& \leqslant\left(\frac{\hat{\ell}-1}{\hat{\ell}}\right) \frac{n}{2}+\frac{\hat{\ell}}{2}=\left(\frac{n(\hat{\ell}-1)+\hat{\ell}^{2}}{n \hat{\ell}}\right) \frac{n}{2},
\end{aligned}
$$

where the last inequality holds because $k \leqslant n / \hat{\ell}+1$ and $i \leqslant \hat{\ell}-1$.

Since $C_{1}(W)+C_{2}(W)=n$, using (4) we know that

$$
\frac{C_{1}(W)}{C_{2}(W)}=\frac{n}{C_{2}(W)}-1 \geqslant 1+\frac{2\left(n-\hat{\ell}^{2}\right)}{n(\hat{\ell}-1)+\hat{\ell}^{2}}>1+\beta,
$$

where the last inequality follows by the choice of $n_{0}$, because $\beta \leqslant 2 / \hat{\ell}$. But this contradicts the $\beta$-balancedness of the coloring $\chi$ of $W$. Therefore, there exists $r \in[\hat{\ell}] \backslash \bigcup_{j=1}^{i} \sigma(j)$ with $C_{2}\left(I_{r}\right) \geqslant k / 2$. Set $\sigma(i+1)=r$. Then

$$
\begin{aligned}
C_{1}(\sigma, i+1) & =C_{1}(\sigma, i)+C_{1}\left(I_{r}\right) \leqslant\left(C_{2}(\sigma, i)+k\right)+\left(\frac{n}{\hat{\ell}}+1-\frac{k}{2}\right) \\
& =\left(C_{2}(\sigma, i)+\frac{k}{2}\right)+\frac{n}{\hat{\ell}}+1 \leqslant C_{2}(\sigma, i+1)+\frac{n}{\hat{\ell}}+1 .
\end{aligned}
$$

Since $C_{1}(\sigma, i+1) \geqslant C_{2}(\sigma, i+1)-(n / \hat{\ell}+1)$, we conclude from the lat inequality that $\left|C_{1}(\sigma, i+1)-C_{2}(\sigma, i+1)\right| \leqslant n / \hat{\ell}+1$.

Let $H=(W, E)$ be a graph with $W=\left\{w_{1}, \ldots, w_{n}\right\}$ and let $\chi: W \rightarrow[2]$ be a coloring of $W$. Consider a partition of $W$ into a set of intervals $\mathcal{I}=\left\{I_{1}, \ldots, I_{\hat{\ell}}\right\}$. We define $C_{i}(I, \sigma, a, b)=\sum_{j=a}^{b} C_{i}\left(I_{\sigma(j)}\right)$ for $i=1,2$. If it is clear what partition we are considering then we write $C_{i}(\sigma, a, b)$ for simplicity.

Corollary 2.10. For every integer $\hat{\ell} \geqslant 1$ there exists $n_{0}$ such that if $H=(W, E)$ is a graph with $W=\left\{w_{1}, \ldots, w_{n}\right\}$ with $n \geqslant n_{0}$, then every $\beta$-balanced 2 -coloring $\chi$ of $W$ with $\beta \leqslant 2 / \hat{\ell}$, and every partition of $W$ into intervals $I_{1}, \ldots, I_{\hat{\ell}}$ with sizes $\left|I_{1}\right| \leqslant \ldots \leqslant\left|I_{\hat{\ell}}\right| \leqslant\left|I_{1}\right|+1$ there exists a permutation $\sigma:[\hat{\ell}] \rightarrow[\hat{\ell}]$ such that for every pair of integers $1 \leqslant a<b \leqslant \hat{\ell}$,

$$
\left|C_{1}(\sigma, a, b)-C_{2}(\sigma, a, b)\right| \leqslant 2\left(\frac{n}{\hat{\ell}}+1\right) .
$$

Proof. Fix $\hat{\ell} \geqslant 1$ and let $n_{0}$ be obtained from Lemma 2.9 applied with $\hat{\ell}$. Let $H=(W, E)$ be a graph with $W=\left\{w_{1}, \ldots, w_{n}\right\}$ with $n \geqslant n_{0}$. Now fix a $\beta$-balanced 2-coloring $\chi$ of $W$ and a partition of $W$ into intervals $I_{1}, \ldots, I_{\hat{\ell}}$ with $\left|I_{1}\right| \leqslant \ldots \leqslant\left|I_{\hat{\ell}}\right| \leqslant\left|I_{1}\right|+1$, where $\beta \leqslant 2 / \hat{\ell}$. 
Let $\sigma$ be the permutation given by Lemma 2.9. Fix integers $1 \leqslant a<b \leqslant \hat{\ell}$ and suppose w.l.o.g. that $C_{1}(\sigma, a, b) \geqslant C_{2}(\sigma, a, b)$. Therefore

$$
\begin{aligned}
C_{1}(\sigma, a, b) & =C_{1}(\sigma, b)-C_{1}(\sigma, a-1) \\
& \leqslant\left(C_{2}(\sigma, b)+n / \hat{\ell}+1\right)-\left(C_{2}(\sigma, a-1)-(n / \hat{\ell}+1)\right) \\
& =C_{2}(\sigma, a, b)+2(n / \hat{\ell}+1) .
\end{aligned}
$$

The next result, the main result of this subsection, guarantees the local balancedness that we need.

Lemma 2.11. For every $\xi>0$ and every integer $\hat{\ell} \geqslant 1$ there exists $n_{0}$ such that if $H=(W, E)$ is a graph with $W=\left\{w_{1}, \ldots, w_{n}\right\}$ with $n \geqslant n_{0}$, then for every $\beta$-balanced 2-coloring $\chi$ of $W$ with $\beta \leqslant 2 / \hat{\ell}$, and every partition of $W$ into intervals $I_{1}, \ldots, I_{\hat{\ell}}$ with $\left|I_{1}\right| \leqslant \ldots \leqslant\left|I_{\hat{\ell}}\right| \leqslant\left|I_{1}\right|+1$ there exists a permutation $\sigma:[\hat{\ell}] \rightarrow[\hat{\ell}]$ such that for every pair of integers $1 \leqslant a<b \leqslant \hat{\ell}$ with $b-a \geqslant 7 / \xi$, we have

$$
\left|C_{1}(\sigma, a, b)-C_{2}(\sigma, a, b)\right| \leqslant \xi C_{2}(\sigma, a, b),
$$

Proof. Fix constants $\xi>0, \hat{\ell} \geqslant 1$ and let $n_{0}^{\prime}$ be obtained by Corollary 2.10 applied with $\hat{\ell}$. Let $H=(W, E)$ be a graph with vertex set $W=\left\{w_{1}, \ldots, w_{n}\right\}$ for

$$
n \geqslant n_{0}=\max \left\{n_{0}^{\prime},((4+2 \xi) /(3-2 \xi)) \hat{\ell}\right\}
$$

and fix a $\beta$-balanced 2-coloring $\chi$ of $W$ and a partition of $W$ into intervals $I_{1}, \ldots, I_{\hat{\ell}}$ with $\left|I_{1}\right| \leqslant \ldots \leqslant\left|I_{\hat{\ell}}\right| \leqslant\left|I_{1}\right|+1$ where $\beta \leqslant 2 / \hat{\ell}$.

Let $\sigma$ be the permutation given by Corolary 2.10. Fix integers $1 \leqslant a<b \leqslant \hat{\ell}$ such that $b-a>7 / \xi$. Note that, by Corollary 2.10,

$$
\left|C_{1}(\sigma, a, b)-C_{2}(\sigma, a, b)\right| \leqslant 2(n / \hat{\ell}+1) .
$$

The above inequality and the fact that $C_{1}(\sigma, a, b)+C_{2}(\sigma, a, b) \geqslant(b-a)(n / \hat{\ell})$ implies

$$
C_{2}(\sigma, a, b) \geqslant\left(\frac{b-a}{2}\right) \frac{n}{\hat{\ell}}-(n / \hat{\ell}+1) .
$$

By the choice of $a, b$ and $n_{0}$, we have

$$
C_{2}(\sigma, a, b) \geqslant(2 / \xi)(n / \hat{\ell}+1) .
$$

Putting inequalities (6) and (7) together we conclude the proof. 


\section{§3. Proof of the MAIN Result}

Before going into the details of the proof of Theorem 1.3 we give some brief overview discussing the main ideas of the proof and explaining how to connect the results of Section 2 .

Overview of the proof of Theorem 1.3. For every $\gamma>0$ and sufficiently large $n$, given an arbitrary edge coloring of $K_{N}$ with 3 colors for $N=(2+\gamma) n$ we want to prove that if $H$ is a $(\beta, \Delta)$-balanced graph on $n$ vertices, then we always find a monochromatic copy of $H$ in $K_{N}$.

The strategy to prove Theorem 1.3 is to apply the Embedding Lemma (Lemma 2.3) to find the desired copy of $H$ in $K_{N}$. In order to do this we use Lemma 2.8 to find a monochromatic subgraph $G$ of $K_{N}$ composed of sufficiently dense regular pairs. So, using Facts 2.4 and 2.5 it is easy to see that deleting some vertices of $G$ we can find a monochromatic graph $G^{\prime} \subset G$ which has a regular partition containing super-regular pairs covering $(1+o(1)) n$ vertices.

In the second part of the proof we carefully construct a partition of $V(H)$ and, since $H$ has small bandwidth, we make use of Lemma 2.11 to show that this partition is compatible with the partition of $G^{\prime}$. Then, we can apply the Embedding Lemma to find the monochromatic copy of $H$, concluding the proof.

Proof of Theorem 1.3. Let $\gamma>0$ and $\Delta \geqslant 1$ be given. Lemma 2.8 applied with $\gamma$ gives $\varepsilon_{0}$. Next we apply Lemma 2.3 with $d=1 / 4$ and $\Delta$ to get $\varepsilon_{1}$. Set

$$
\varepsilon=\min \left\{\varepsilon_{0}, \varepsilon_{1} / 2, \gamma / 19\right\}
$$

Since $\varepsilon \leqslant \varepsilon_{0}$, Lemma 2.8 gives to us a natural number $K_{0}$. Fix $\xi=\gamma / 304$ and let $n_{0}$ be obtained by an application of Lemma 2.11 with parameters $\xi$ and $K_{0}$. Set

$$
\beta=\varepsilon \xi(1+2 \xi) / 36 \Delta^{2} K_{0}^{2} .
$$

Let $H=\left(W, E_{H}\right)$ be a balanced $(\beta, \Delta)$-graph on $n$ vertices. Now put $N=\lfloor(2+\gamma) n\rfloor$, where $N \geqslant \max \left\{n_{0}, K_{0}\right\}$. Consider an arbitrary coloring $\chi_{K_{N}}: E\left(K_{N}\right) \rightarrow[3]$ of the edges of $K_{N}$. We want to show that every such coloring yields a monochromatic copy of $H$.

Partitioning the vertices of $K_{N}$. Next we find a monochromatic and sufficiently regular subgraph $G^{\prime}$ of $K_{N}$. By Lemma 2.8, there are a color (say color 1 ), integers $\ell, \ell^{\prime}, k$ with $\ell, \ell^{\prime} \leqslant k \leqslant K_{0}$ and $\ell \geqslant(1-\gamma / 4) k / 4$, a tree $T$ on vertex set $\left\{x_{1}, \ldots, x_{\ell}, y_{1}, \ldots, y_{\ell}, z_{1}, \ldots, z_{\ell^{\prime}}\right\}$ containing a matching $M$ with edge set $E_{M}=\left\{x_{i} y_{i}: i=1, \ldots, \ell\right\}$ with an even distance in $T$ between any $x_{i}$ and $x_{j}$ for all $i$ and $j$, such that there exists a partition $\left(V_{i}\right)_{i \in[k]}$ of $V=V\left(K_{N}\right)$ such that $K_{N}^{1}$ is $(\varepsilon, 1 / 3)$-regular on $T$ and $\left|V_{1}\right|=\ldots=\left|V_{k}\right|=m$, where 
$m \geqslant(1-\varepsilon) N / k$. Let $G_{T}$ be the subgraph of $K_{N}^{1}$ induced by the classes in $\left(V_{i}\right)_{i \in[k]}$ corresponding to the vertices of $T$.

In order to apply the Embedding Lemma, we need the classes of $G_{T}$ that correspond to the matching edges to form super-regular pairs and the other pairs of classes should be sufficiently regular. We can ensure this by deleting some vertices of $G_{T}$. In fact, applying Fact 2.5 and, after that, Fact 2.4, it is easy to see that we find a subgraph $G^{\prime} \subset G_{T}$ with classes $A_{1}, \ldots, A_{\ell}, B_{1}, \ldots, B_{\ell}, C_{1}, \ldots, C_{\ell^{\prime}}$ of size at least $(1-\varepsilon) m$ corresponding, respectively, to the vertices $x_{1}, \ldots, x_{\ell}, y_{1}, \ldots, y_{\ell}, z_{1}, \ldots, z_{\ell^{\prime}}$ of the tree $T$, such that the bipartite graphs induced by $A_{i}$ and $B_{i}$ are $(2 \varepsilon, 1 / 3-\varepsilon)$-super-regular and the bipartite graphs induced by all the other pairs are $(2 \varepsilon, 1 / 3-\varepsilon)$-regular. Furthermore, let $D_{\min }$ be the set with the smallest cardinality among the sets in $A_{1}, \ldots, A_{\ell}, B_{1}, \ldots, B_{\ell}, C_{1}, \ldots, C_{\ell^{\prime}}$. Since $\varepsilon \leqslant \gamma / 19, N=\lfloor(2+\gamma) n\rfloor, m \geqslant(1-\varepsilon) N / k$ and $\ell \geqslant(1-\gamma / 4) k / 4$, one can see that

$$
\left|D_{\min }\right| \geqslant(1+\gamma / 152) n / 2 \ell \text {. }
$$

Partitioning the vertices of $H$. Now it is time to construct a partition of $W$ ready for the application of Lemma 2.3. Since $H$ is a balanced $(\beta, \Delta)$-graph, there exists a coloring $\chi_{H}: V(H) \rightarrow[2]$ such that ||$\chi^{-1}(1)|-| \chi^{-1}(2)|| \leqslant \beta\left|\chi^{-1}(2)\right|$.

Let $w_{1}, \ldots, w_{n}$ be an ordering of $W$ such that $|i-j| \leqslant \beta n$ for every $w_{i} w_{j} \in E_{H}$ and let $\hat{\ell}$ be the smallest integer dividing $n$ with $\hat{\ell} \geqslant\left(7 K_{0} / \xi\right)+\ell \geqslant \ell(7 / \xi+1)$. Consider the partition of $V(H)$ into intervals $I_{1}, \ldots, I_{\hat{\ell}}$ with $\left|I_{1}\right|=\ldots=\left|I_{\hat{\ell}}\right|=n / \hat{\ell}$ taking this ordering into account, i.e., $I_{i}=w_{(i-1) n / \hat{\ell}+1}, \ldots, w_{i n / \hat{\ell}}$ for $i=1, \ldots, \hat{\ell}$. By Lemma 2.11 , since $\beta \leqslant 2 / \hat{\ell}$, there exists a permutation $\sigma:[\hat{\ell}] \rightarrow[\hat{\ell}]$ such that

$$
\left|C_{1}(\sigma, a, b)-C_{2}(\sigma, a, b)\right| \leqslant \xi C_{2}(\sigma, a, b)
$$

for all integers $1 \leqslant a<b \leqslant \hat{\ell}$ with $b-a \geqslant 7 / \xi$. Define $a_{i}=(i-1) \hat{\ell} / \ell+1$ and $b_{i}=i \hat{\ell} / \ell$ and consider the blocks $J_{i}=\left\{I_{\sigma\left(a_{i}\right)}, I_{\sigma\left(a_{i}+1\right)}, \ldots, I_{\sigma\left(b_{i}\right)}\right\}$ for $i=1, \ldots, \ell$. We write $C_{1}\left(J_{i}\right)$ for $C_{1}\left(\sigma, a_{i}, b_{i}\right)$ and $C_{2}\left(J_{i}\right)$ for $C_{2}\left(\sigma, a_{i}, b_{i}\right)$. Thus, for $i=1, \ldots, \ell$, since $b_{i}-a_{i}=\hat{\ell} / \ell+1 \geqslant 7 / \xi$, we have

$$
\left|C_{1}\left(J_{i}\right)-C_{2}\left(J_{i}\right)\right| \leqslant \xi C_{2}\left(J_{i}\right),
$$

Recall we have found a tree $T$ on vertex set $\left\{x_{1}, \ldots, x_{\ell}, y_{1}, \ldots, y_{\ell}, z_{1}, \ldots, z_{\ell^{\prime}}\right\}$ containing matching edges $E_{M}=\left\{x_{i} y_{i}: i=1, \ldots, \ell\right\}$ such that the distance in $T$ between any $x_{i}$ and $x_{j}$ for all $i$ and $j$ is even. Our partition of $W$ will be composed of clusters $X_{1}, \ldots, X_{\ell}, Y_{1}, \ldots, Y_{\ell}, Z_{1}, \ldots, Z_{\ell^{\prime}}$ corresponding to $x_{1}, \ldots, x_{\ell}, y_{1}, \ldots, y_{\ell}, z_{1}, \ldots, z_{\ell^{\prime}}$.

For every $i=1, \ldots, \ell$, we will put most of the vertices of $J_{i}$ in the clusters $X_{i}$ and $Y_{i}$, depending on the color they received from $\chi_{H}$. The remaining vertices will be distributed in order to make it possible to "walk" between the matching clusters. 
We divide each interval $I_{i}$ in two parts. The first one, called link of $I_{i}$, is denoted by $L_{i}$. The links are responsible to make the connections between the matching clusters. For the last interval, we set $L_{\hat{\ell}}=\varnothing$. For $1 \leqslant i \leqslant \hat{\ell}-1$, if $I_{i}$ and $I_{i+1}$ are in the same block $J_{r}$, then $L_{i}=\varnothing$.

Suppose that $I_{i} \in J_{r}$ and $I_{i+1} \in J_{s}$ with $r \neq s$ and $1 \leqslant i \leqslant \hat{\ell}-1$. Let $P_{T}(r, s)$ be the path of $T$ between $x_{r}$ and $x_{s}$ and consider the path $P_{T}^{\text {int }}(r, s) \subset P_{T}(r, s)$ obtained by excluding the vertices of the set $\left\{x_{r}, y_{r}, x_{s}, y_{s}\right\}$ from $P_{T}(r, s)$, i.e., $P_{T}^{\text {int }}(r, s)$ is the "internal" part of the path of $T$ that one should use to reach $x_{s}$ from $x_{r}$. For a lighter notation set $t_{r, s}=\left|P_{T}^{\text {int }}(r, s)\right|$. We divide the $\left(t_{r, s}+1\right) \beta n$ last vertices of $I_{i}$ in $t_{r, s}+1$ "pieces" of size $\beta n$, respecting their sequence in the interval, where the $j$-th piece is denoted by $L_{i}(j)$ for $1 \leqslant j \leqslant t_{r, s}+1$, that is,

$$
L_{i}(j)=w_{\left(i-\left(t_{r, s}+2-j\right) \beta \hat{\ell}\right) n / \hat{\ell}+1}, \ldots, w_{\left(i-\left(t_{r, s}+1-j\right) \beta \hat{\ell}\right) n / \hat{\ell}}
$$

We put $L_{i}=\left\{L_{i}(1), \ldots, L_{i}\left(t_{r, s}\right), L_{i}\left(t_{r, s}+1\right)\right\}$.

Since we have described the links, we can now define the main part of the intervals. We define $\mathrm{KE}_{i}=I_{i} \backslash L_{i}$ as the kernel of the interval $I_{i}$, which will be placed on the matching clusters $X_{i}$ and $Y_{i}$.

We have to construct the clusters that will compose the partition of $H$. Initially, let each cluster in $\left\{X_{1}, \ldots, X_{\ell}, Y_{1}, \ldots, Y_{\ell}, Z_{1}, \ldots, Z_{\ell^{\prime}}\right\}$ be empty. Consider the block $J_{i}$ for every $1 \leqslant i \leqslant \ell$. For each interval $I_{p} \in J_{i}$ we include in $X_{i}$ all the vertices $w$ of the kernel $\mathrm{KE}_{p}$ with $\chi_{H}(w)=1$ and we include in $Y_{i}$ all the vertices $w$ of $\mathrm{KE}_{p}$ with $\chi_{H}(w)=2$.

The next step is to accommodate all the links. Consider the interval $I_{i}$ for $1 \leqslant i \leqslant \hat{\ell}-1$ and assume that $I_{i}$ is in $J_{r}$ and $I_{i+1}$ is in $J_{s}$ with $r \neq s$, otherwise the link we are looking for is empty and there is nothing to do. Denote the internal path $P_{T}^{\text {int }}(r, s)$ of $P_{T}(r, s)$ by $\left\{u_{1}, \ldots, u_{t_{r, s}}\right\}$ and let $u_{0}$ and $u_{t_{r, s}+1}$ be, respectively, the vertices of $T$ connected to $u_{1}$ and $\mathrm{t} u_{t_{r, s}}$ in $P_{T}(r, s)$.

Now we will show how it is possible to "walk" between the matching clusters. note that $u_{0}$ can be either $x_{r}$ or $y_{r}$. Without loss of generality we assume that $u_{0}=x_{r}$. For $1 \leqslant j \leqslant t_{r, s}+1$, we put the vertices $w$ of $L_{i}(j)$ with $\chi_{H}(w)=1$ in the corresponding class of $u_{j-1}$ if $j$ is even, and in the corresponding class of $u_{j}$ if $j$ is odd. For those $w$ with $\chi_{H}(w)=2$, we do the other way around, i.e., we put them in the corresponding class of $u_{j}$ if $j$ is even, and in the corresponding class of $u_{j-1}$ if $j$ is odd. Since $x_{i}$ and $x_{j}$ are at an even distance for all $1 \leqslant i<j \leqslant \ell$ and the links have size $\beta n$, we know that there is no edges inside the clusters and if there is an edge between two clusters, then the corresponding edge is present in $T$. 
Applying the Embedding Lemma. Here we will show that the vertex partition of $W$ is $\left(2 \varepsilon_{1}, T, M\right)$-compatible with the partition of $V\left(G^{\prime}\right)$ we constructed before. Thus, we can apply the Embedding Lemma to find the desired monochromatic copy of $H$ in $K_{N}$.

The first step is to bound by above the size of each cluster in the partition

$$
\left\{X_{1}, \ldots, X_{\ell}, Y_{1}, \ldots, Y_{\ell}, Z_{1}, \ldots, Z_{\ell^{\prime}}\right\}
$$

of $W$. Note that, for every $1 \leqslant i \leqslant \ell$, we have $C_{1}\left(J_{i}\right)+C_{2}\left(J_{i}\right)=n / \ell$. Using this fact and $(9)$ one can easily obtain that, for every $1 \leqslant i \leqslant \ell$,

$$
(1-\xi) \frac{n}{2 \ell} \leqslant C_{1}\left(J_{i}\right), C_{2}\left(J_{i}\right) \leqslant(1+\xi) \frac{n}{2 \ell} .
$$

By the construction, every set $X_{i}$ (resp. $Y_{i}$ ) is composed only of vertices $v$ with $\chi(v)=1$ $(\chi(v)=2)$. Furthermore, these vertices can come from one kernel and at most two pieces of each link. Then,

$$
\left|X_{i}\right|,\left|Y_{i}\right| \leqslant(1+\xi) \frac{n}{2 \ell}+2 \hat{\ell} \beta n=(1+\xi+4 \ell \hat{\ell} \beta) \frac{n}{2 \ell} \leqslant\left|D_{\min }\right|,
$$

where the last inequality follows by inequality (8) and the choice of $\xi, \beta$ and $\hat{\ell}$.

For the clusters $Z_{i}$, for $1 \leqslant i \leqslant \ell^{\prime}$, we know that they are composed only of vertices in at most two pieces of each link. Thus,

$$
\left|Z_{i}\right| \leqslant 2 \hat{\ell} \beta n=(4 \ell \hat{\ell} \beta) \frac{n}{2 \ell} \leqslant \frac{\varepsilon}{\Delta^{2}}\left|D_{\min }\right|,
$$

where the last inequality follows by inequality (8) and the choice of $\beta$ and $\hat{\ell}$.

Now we can check that the partitions of $W$ and $V\left(G^{\prime}\right)$ are compatible. Based on Definition 2.2 we define the sets $U_{j}$ and $U_{j}^{\prime}$ for $1 \leqslant j \leqslant 2 \ell+\ell^{\prime}$ with respect to the partition $\left\{X_{1}, \ldots, X_{\ell}, Y_{1}, \ldots, Y_{\ell}, Z_{1}, \ldots, Z_{\ell^{\prime}}\right\}$ of $W$. Define $W_{j}=X_{j}$ if $1 \leqslant j \leqslant \ell, W_{j}=Y_{j-\ell}$ if $\ell+1 \leqslant j \leqslant 2 \ell$, and $W_{j}=Z_{j-2 \ell}$ if $2 \ell+1 \leqslant j \leqslant 2 \ell+\ell^{\prime}$. Then, we will verify that the four conditions of Definition 2.2 hold:

( $i$ ) By the construction of the partition of $W$, if there is an edge between two clusters, then the corresponding edge is present in $T$.

(ii) Owing to (8) every set $D$ in the partition $\left\{A_{1}, \ldots, A_{\ell}, B_{1}, \ldots, B_{\ell}, C_{1}, \ldots, C_{\ell^{\prime}}\right\}$ of $V\left(G^{\prime}\right)$ has size $|D| \geqslant(1+\gamma / 152) n / 2 \ell$. So, inequalities (11) and (12) show that condition (II) holds.

(iii) Fix $1 \leqslant j \leqslant 2 \ell+\ell^{\prime}$. Define $U_{j}$ as the set of vertices of $W_{j}$ with neighbors in some $W_{k}$ with $j \neq k$ and $\{j, k\} \notin M$. We divide in two cases:

(a) $2 \ell+1 \leqslant j \leqslant 2 \ell+\ell^{\prime}$ : We have $U_{j}=Z_{j-2 \ell}$. By $(12),\left|U_{j}\right| \leqslant \varepsilon\left|D_{\min }\right| / \Delta$.

(b) $1 \leqslant j \leqslant 2 \ell$ : In this case, $U_{j}$ is composed only of neighbors of vertices in exactly one set of $\left\{Z_{1}, \ldots, Z_{\ell^{\prime}}\right\}$. Thus, since $\Delta$ if the maximum degree of $H$, by (12), we conclude that $\left|U_{j}\right| \leqslant \varepsilon\left|D_{\min }\right| / \Delta$. 
Thus, for every $j=1, \ldots, 2 \ell+\ell^{\prime}$ we have

$$
\left|U_{j}\right| \leqslant \frac{\varepsilon}{\Delta}\left|D_{\min }\right|
$$

which shows that condition (III) holds.

(iv) Define the set $U_{j}^{\prime}=N_{H}(U) \cap\left(W_{j} \backslash U\right)$, where $U=\bigcup_{i=1}^{2 \ell+\ell^{\prime}} U_{i}$. Consider the following cases.

(a) $2 \ell+1 \leqslant j \leqslant 2 \ell+\ell^{\prime}$ : Note that since every vertex of $Z_{j-2 \ell}$ belongs to $U_{j}$, we have $U_{j}^{\prime}=\varnothing$. Thus, it is obvious that $\left|U_{j}^{\prime}\right| \leqslant\left|D_{\min }\right|$.

(b) $\ell+1 \leqslant j \leqslant 2 \ell$ : Here, $U_{j}^{\prime} \subset W_{j}=Y_{j-\ell}$. Then, $U_{j}^{\prime}$ is composed only of neighbors of $U_{j-\ell} \subset X_{j-\ell}$. Then, using (13), we have $\left|U_{j}^{\prime}\right| \leqslant \Delta\left|U_{j-\ell}\right| \leqslant \varepsilon\left|D_{\min }\right|$.

(c) $1 \leqslant i \leqslant \ell$ : This case is analogous to case (b).

Since we proved that the four conditions of Definition 2.2 hold, the partition

$$
\left\{X_{1}, \ldots, X_{\ell}, Y_{1}, \ldots, Y_{\ell}, Z_{1}, \ldots, Z_{\ell^{\prime}}\right\}
$$

of $W$ is $(2 \varepsilon, T, M)$-compatible (then, it is clearly $\left(\varepsilon_{1}, T, M\right)$-compatible) with

$$
\left\{A_{1}, \ldots, A_{\ell}, B_{1}, \ldots, B_{\ell}, C_{1}, \ldots, C_{\ell^{\prime}}\right\}
$$

which is a partition of $V\left(G^{\prime}\right)$. Then, by Lemma 2.3, we conclude that $H \subset G^{\prime}$. This finishes the proof, since $G^{\prime}$ is a monochromatic subgraph of $K_{N}$.

\section{§4. Sketch of the Proof of Theorem 1.2}

We show that for every $\gamma>0$ and natural number $\Delta$, there exists a constant $\beta>0$ such that for every sufficiently large $(\beta, \Delta)$-graph $H$ with a proper 2-coloring $\chi_{H}: V(H) \rightarrow[2]$ where $t_{1}=\left|\chi_{H}^{-1}(1)\right|$ and $t_{2}=\left|\chi_{H}^{-1}(2)\right|$, with $t_{1} \leqslant t_{2}$, we can find a monochromatic copy of $H$ in every edge coloring of $E\left(K_{N}\right)$ with $N=(1+\gamma) \max \left\{2 t_{1}+t_{2}, 2 t_{2}\right\}$. Let $H$ be such a graph and assume $2 t_{1} \geqslant t_{2}$ (the complementary case can be solved in a similar way).

The proof of Theorem 1.2 is very similar to the proof of Theorem 1.3. Here we also embed $H$ in parts, considering a partition of a monochromatic subgraph $G$ of $K_{N}$. The partition we need is composed of a special cluster $W$ and clusters $X_{1}, Y_{1}, \ldots, X_{m}, Y_{m}$ corresponding to a "large" matching $M$ with matching edges $E_{M}=\left\{x_{i}, y_{i}: i=1, \ldots, m\right\}$ such that for every $i=1, \ldots, m$ the pairs $\left\{X_{i}, Y_{i}\right\}$ are super-regular and the pairs $\left\{X_{i}, W\right\}$ are regular. The special cluster $W$ is needed to allow us to "walk" between the clusters $X_{1}, Y_{1}, \ldots, X_{m}, Y_{m}$.

The problem in the preparation of the host monochromatic graph $G$ is the fact that $H$ is not as balanced as it is in the setup of Theorem 1.3. So, in order to embed $H$ in $G$ we need that $\left|Y_{i}\right| /\left|X_{i}\right|=t_{2} / t_{1}$. Fortunately, by [13, Theorem 3], since $t_{2} / t_{1} \leqslant 2$ in the case we 
are considering, we can find such a monochromatic graph $G$. Using Fact 2.5 we can easily make the matching pairs super-regular.

Now we have to prepare the graph $H$ for the embedding. We consider the ordering of its vertices respecting the bandwidth condition and divide the set of vertices into intervals. Thus, we can find a permutation of such intervals such that blocks of intervals fit into the super-regular pairs of $G$. Then, using few vertices we can "walk" from one super-regular pair to another as done in the proof of Theorem 1.3 and we are done.

\section{REFERENCES}

[1] P. Allen, G. Brightwell, and J. Skokan, Ramsey-goodness - and otherwise, Combinatorica 33 (2013), no. 2, 125-160. MR3071850 1

[2] F. S. Benevides and J. Skokan, The 3-colored Ramsey number of even cycles, J. Combin. Theory Ser. B 99 (2009), no. 4, 690-708. MR2518202 (2010h:05186) 11

[3] J. Böttcher, Embedding large graphs - the Bollobás-Komlós conjecture and beyond, Ph.D. Thesis, 2009. $\uparrow 2.1,2.3$

[4] J. Böttcher, P. Heinig, and A. Taraz, Embedding into bipartite graphs, SIAM J. Discrete Math. 24 (2010), no. 4, 1215-1233. MR2735919 (2012a:05241) $\uparrow 2.3$

[5] J. Böttcher, K. P. Pruessmann, A. Taraz, and A. Würfl, Bandwidth, expansion, treewidth, separators and universality for bounded-degree graphs, European J. Combin. 31 (2010), no. 5, 1217-1227. MR2644412 (2012a:05091) 11

[6] R. J. Faudree and R. H. Schelp, Path Ramsey numbers in multicolorings, J. Combinatorial Theory Ser. B 19 (1975), no. 2, 150-160. MR0412023 (54 \#152) 11

[7] A. Figaj and T. Łuczak, The Ramsey number for a triple of long even cycles, J. Combin. Theory Ser. B 97 (2007), no. 4, 584-596. MR2325798 (2008c:05119) $\uparrow 1,2.2$

[8] L. Gerencsér and A. Gyárfás, On Ramsey-type problems, Ann. Univ. Sci. Budapest. Eötvös Sect. Math. 10 (1967), 167-170. MR0239997 (39 \#1351) 11

[9] R. L. Graham, B. L. Rothschild, and J. H. Spencer, Ramsey theory, Second, Wiley-Interscience Series in Discrete Mathematics and Optimization, John Wiley \& Sons Inc., New York, 1990. A Wiley-Interscience Publication. MR1044995 (90m:05003) $\uparrow 1$

[10] A. Gyárfás, M. Ruszinkó, G. N. Sárközy, and E. Szemerédi, Three-color Ramsey numbers for paths, Combinatorica 27 (2007), no. 1, 35-69. MR2310787 (2008b:05110) ^1, 2.2, 2.2

[11] — Tripartite Ramsey numbers for paths, J. Graph Theory 55 (2007), no. 2, 164-174. MR2316280 (2008b:05111) $\uparrow 2.2$

[12] P. E. Haxell, T. Łuczak, Y. Peng, V. Rödl, A. Ruciński, M. Simonovits, and J. Skokan, The Ramsey number of hypergraph cycles. I, J. Combin. Theory Ser. A 113 (2006), no. 1, 67-83. MR2192770 (2006i:05110) $\uparrow 2.2$

[13] P. E. Haxell, T. Łuczak, and P. W. Tingley, Ramsey numbers for trees of small maximum degree, Combinatorica 22 (2002), no. 2, 287-320. Special issue: Paul Erdős and his mathematics. MR1909088 (2003d:05141) $\uparrow 1,1,1,4$ 
[14] J. Komlós and M. Simonovits, Szemerédi's regularity lemma and its applications in graph theory, Combinatorics, Paul Erdős is eighty, Vol. 2 (Keszthely, 1993), 1996, pp. 295-352. MR1395865 (97d:05172) $\uparrow 2.1$

[15] J. Komlós, G. N. Sárközy, and E. Szemerédi, Blow-up lemma, Combinatorica 17 (1997), no. 1, 109123. MR1466579 (99b:05083) $\uparrow 2.1$

[16] J. Komlós, G. N. Sárközy, and E. Szemerédi, An algorithmic version of the blow-up lemma, Random Structures Algorithms 12 (1998), no. 3, 297-312. MR1635264 (99h:05113) 22.1

[17] J. Komlós, A. Shokoufandeh, M. Simonovits, and E. Szemerédi, The regularity lemma and its applications in graph theory, Theoretical aspects of computer science (Tehran, 2000), 2002, pp. 84-112. MR1966181 (2004d:05106) $\uparrow 2.1$

[18] T. Łuczak, $R\left(C_{n}, C_{n}, C_{n}\right) \leqslant(4+o(1)) n$, J. Combin. Theory Ser. B 75 (1999), no. 2, 174-187. MR1676887 (2000b:05096) $\uparrow 2.2$

[19] S. P. Radziszowski, Small Ramsey numbers, Electron. J. Combin. 1 (1994 (Lastest update: 2011)), Dynamic Survey 1, 84 pp. (electronic). MR1670625 (99k:05117) $\uparrow 1$

[20] V. Rödl, A. Ruciński, and A. Taraz, Hypergraph packing and graph embedding, Combin. Probab. Comput. 8 (1999), no. 4, 363-376. Random graphs and combinatorial structures (Oberwolfach, 1997). MR1723649 (2000m:05164) $\uparrow 2.1$

[21] V. Rödl and A. Ruciński, Perfect matchings in $\epsilon$-regular graphs and the blow-up lemma, Combinatorica 19 (1999), no. 3, 437-452. MR1723256 (2000j:05100) $\uparrow 2.1$

[22] E. Szemerédi, Regular partitions of graphs, Problèmes combinatoires et théorie des graphes (Colloq. Internat. CNRS, Univ. Orsay, Orsay, 1976), 1978, pp. 399-401. MR540024 (81i:05095) 22

Instituto de Matemática e Estatística, Universidade de São Paulo, São Paulo, Brazil E-mail address: mota@ime.usp.br

Computer Science Department, Worcester Polytechnic Institute, Worcester, USA and Alfréd Rényi Institute of Mathematics, Hungarian Academy of Sciences, Budapest, HunGARY

E-mail address: gsarkozy@cs.wpi.edu

Fachbereich Mathematik, Universität Hamburg, Hamburg, Germany

E-mail address: schacht@math.uni-hamburg.de

Institut für Mathematik, Technische Universität Hamburg-Harburg, Hamburg, GerMANY

E-mail address: taraz@tuhh.de 